\title{
X-ray Luminosity Functions and Star Formation Rate
}

\author{
H.-J. Grimm ${ }^{1} \dagger$ M. Gilfanov ${ }^{2}$ and R. Sunyaev ${ }^{2}$ \\ ${ }^{1}$ Harvard-Smithsonian Center for Astrophysics, 60 Garden Street, Cambridge, MA, USA \\ email: hgrimm@head.cfa.harvard.edu \\ ${ }^{2}$ Max-Planck-Institut für Astrophysik, Karl-Schwarzschild-Strasse 1, 85471 Garching, Germany
}

\begin{abstract}
I will discuss the connection between the luminosity function of a population of highmass X-ray binaries in a galaxy and the star formation rate in the this galaxy. The understanding of this connection provides on the one hand an independent measure of an important galaxy property, and on the other hand new insights into populations of high-mass X-ray binaries. In particular, observations with the Chandra X-ray telescope are uniquely suited to investigate the $\mathrm{X}$-ray part of this connection and I will present examples of this.
\end{abstract}

Keywords. X-rays:binaries, X-rays:galaxies, galaxies:fundamental parameters.

\section{Introduction}

X-ray binaries can be separated into two different classes according to the mass of the secondary star. Low-mass X-ray binaries (LMXBs) contain black holes or neutron stars as the primary object, and a star of less than $2.5 \mathrm{M}_{\odot}$ as secondary. High-mass X-ray binaries (HMXBs) on the other hand have a secondary of more than $2.5 \mathrm{M}_{\odot}$. In fact, in the Milky Way most secondaries in LMXBs have masses less than $1 \mathrm{M}_{\odot}$, and most HMXBs have secondaries with more than $10 \mathrm{M}_{\odot}$. Only few objects are known to have secondaries in the gap between $1 \mathrm{M}_{\odot}$ and $10 \mathrm{M}_{\odot}$.

The difference in secondary masses results in two important physical distinctions between LMXBs and HMXBs. The first of these concerns the lifetime of the system. Lifetimes of main sequence stars are roughly proportional to $\mathrm{M}_{*}^{-3}$. Thus, the massive stars in HMXBs have shorter lifetimes than the stars in LMXBs by a factor of $\sim 1000$ or more, i.e. $10^{5}$-few times $10^{7}$ years. Due to the short lifetime HMXBs are a good estimator of recent star formation rate. Moreover, the location of HMXBs is close to star formation regions because they live only shortly and also have smaller systemic velocities.

The second distinction is with regard to the mass loss (and thus accretion) mechanism of the secondary star. The stars in LMXBs lose mass to the primary exclusively through Roche lobe overflow. The mass is lost through the inner Lagrange point of the system and forms an accretion disk around the primary object. In HMXBs this mechanism exists as well. But because massive stars have strong stellar winds, the primary can also accrete mass from just the wind without the secondary filling its Roche lobe.

To investigate the relation between high-mass X-ray binaries and star formation rate we need to understand star formation measures. Star formation is measured by various different indicators in different spectral bands. The bands range from radio to UV, the most common being radio flux at $1.4 \mathrm{GHz}$, far-infrared flux, UV flux, and $\mathrm{H}_{\alpha}$. Despite the use of quite different energy bands almost all indicators measure the ionizing photon flux from massive stars $\left(\mathrm{M}>8 \mathrm{M}_{\odot}\right)$ in star forming regions. $\mathrm{H}_{\alpha}$ and UV measure this

$\dagger$ Present address: Harvard-Smithsonian Center for Astrophysics, 60 Garden Street, Cambridge, MA, USA. 

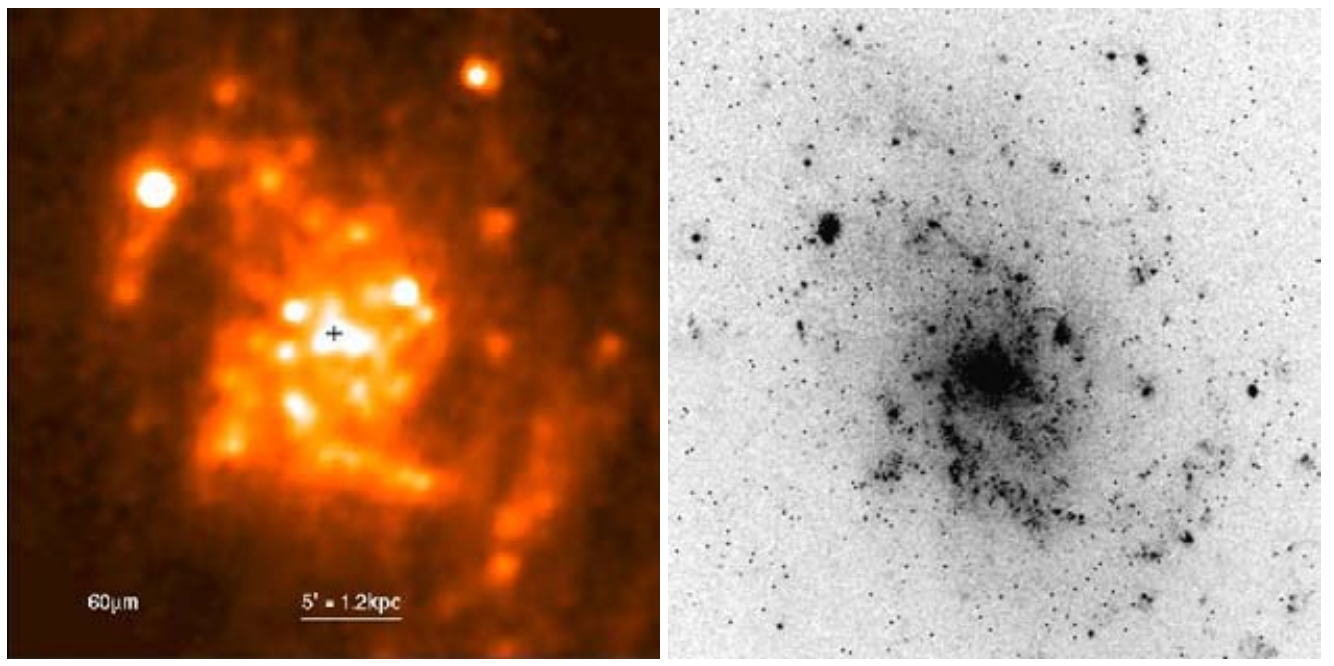

Figure 1. The panels show the galaxy M33 in different spectral bands, 60 micron far-infrared (left) and $\mathrm{H}_{\alpha}$ (right). Both spectral bands are used to measure star formation rate. Despite the different bands (FIR versus UV), these images show the same structure. Both SFR indicators measure the ionizing photon flux from massive stars $\left(\mathrm{M}>8 \mathrm{M}_{\odot}\right)$ in star forming regions. $\mathrm{H}_{\alpha}$ measures this flux directly while FIR measures the reprocessed ionizing flux from dust. Pictures taken from the NED database. Sources: Hippelein et al. (2003) and Cheng et al. (1997).

flux directly while FIR measures the reprocessed ionizing flux from dust. The radio flux measures the thermal emission from HII star formation regions.

The main problem is the conversion from the measured fluxes to actual star formation rates. In measuring the thermal radio flux it is difficult to disentangle the thermal from non-thermal emission. Other indicators suffer from uncertainties in the escape fraction of ionizing photons, dust absorption, and contamination.

\section{Luminosity functions}

X-ray binary luminosity functions have become a diagnostic for populations with the advent of XMM-Newton and particularly Chandra. Observations of other galaxies have the advantage of uniform coverage and known distance for all sources. On the other hand only in the Milky Way, and the Magellanic Clouds, are unique optical counterparts available for identification. Also only in the Milky Way sources with luminosities below $10^{36} \mathrm{erg} \mathrm{s}^{-1}$ are observable. The difficulty with the X-ray binary population of the Milky Way is that the construction of the luminosity function requires knowledge of the mass distribution of the Milky Way to correct for the non-uniform X-ray luminosity coverage (Grimm et al. 2002). In other galaxies it is in general not possible to distinguish between LMXBs and HMXBs.

To investigate the connection between HMXBs and SFR we thus selected galaxies whose X-ray binary population is dominated by HMXBs, i.e. star formation (Grimm et al. 2003). This selection is based on the assumption that HMXBs are related to star formation rate whereas LMXBs are related to the mass of a galaxy. Galaxies that have a sufficiently high value of the ratio star formation rate versus galaxy mass were selected. All the galaxies are of late Hubble type. The luminosity functions cover a range of 4 orders of magnitude in luminosity and a factor of $\sim 40$ in star formation rate. The observed luminosity functions are shown in the left panel of Fig. 2. 

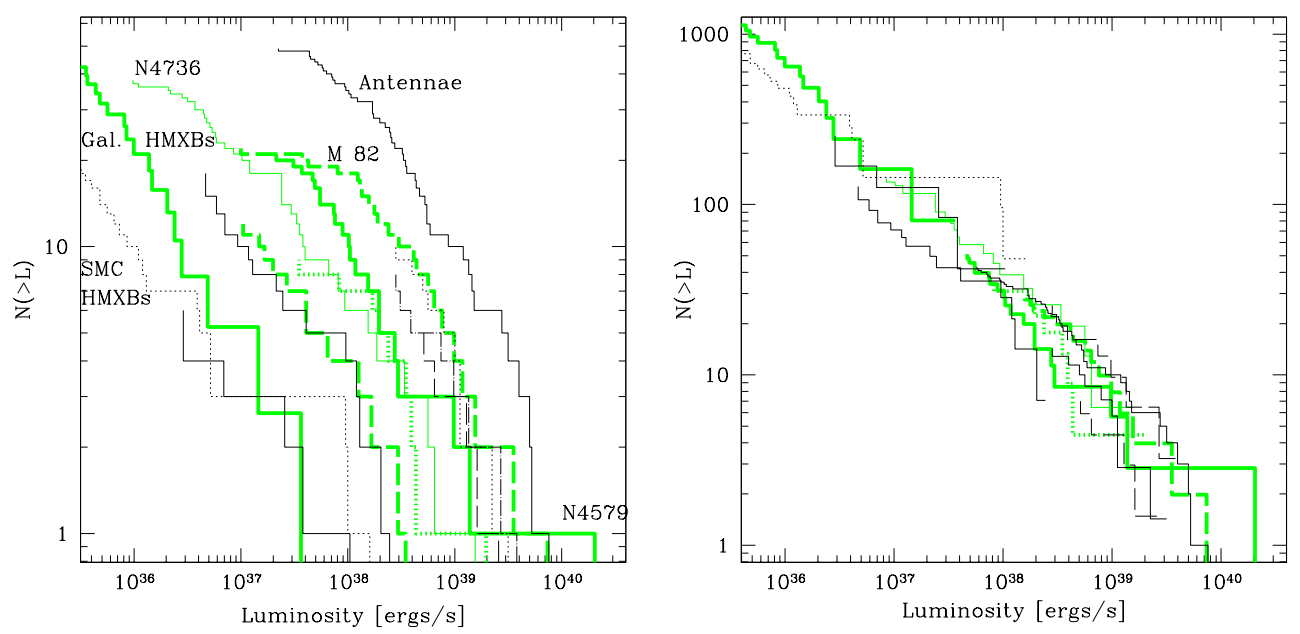

Figure 2. Left: Luminosity functions of various actively star forming galaxies observed with Chandra and of HMXBs in the Milky Way. Only very nearby galaxies have unique optical counterparts. The Milky Way luminosity function is dependent on knowledge of the mass distribution of the Milky Way. Note the wide spread in normalization of the luminosity functions observed by Chandra. Right: Chandra luminosity functions scaled by the ratio of their star formation rates to the star formation rate of the Antennae galaxies.

If one scales the luminosity functions by their respective star formation rates the large spread in normalizations disappears. The scaled luminosity functions fall into a relatively narrow range in the number-luminosity plane. Although there is still some spread at present this can be easily accounted for by uncertainties in the SFR measurement other measurement uncertainties. It is therefore possible to combine the scaled luminosity functions into a single "universal" luminosity function for HMXBs.

The universal luminosity function has the shape of a simple power law. There is an indication for a cutoff at $2 \cdot 10^{40} \mathrm{erg} \mathrm{s}^{-1}$. From the luminosity function the evidence for a cutoff is not significant. However, there is independent evidence for the existence of a cutoff that will be presented below. The universal luminosity function is given by

$$
\frac{d N}{d L_{38}}=3.3 \cdot S F R \cdot L_{38}^{-1.61}, \text { if } L_{38}<200
$$

with $L_{38}=10^{38} L \operatorname{erg~s}^{-1}$.

Surprisingly there are no strong deviations from this power law over the 5 orders of magnitude in luminosity where it has been determined. Although there are considerable uncertainties, especially with star formation rate measurements, the simple shape is unexpected because of the different source types contributing to the luminosity function. At low luminosities the main contributors are Be/X-ray binaries with neutron star primaries. At medium luminosities wind accreting systems with either black hole or neutron star primaries contribute mostly. At high luminosities back holes with supergiant secondaries are the main contributors. At the highest luminosities sources are defined as ULXs. As the exact nature of ULXs is still unknown, it is only possible to state that the smooth extension of the luminosity function to the ULX regime suggests that most of them are part of the X-ray binary population.

The upper end of the luminosity function and the objects that populate this luminosity range has been an area of intense research in recent years. Because of the excellent angular and spectral resolution of Chandra, and XMM, it is possible to identify ULXs in distant 


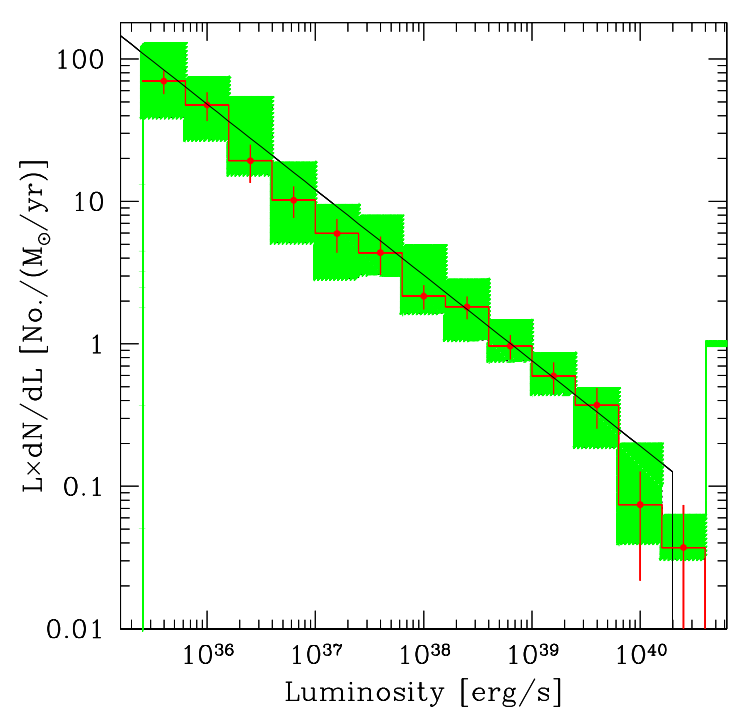

Figure 3. Universal luminosity function of HMXBs. There are no strong deviations from this power law over the 5 orders of magnitude in luminosity where it has been determined. The cutoff at high luminosities is not significant from the luminosity function alone but the relation of the total X-ray versus SFR requires the existence of a cutoff in the range of the indicated luminosities.

galaxies as individual objects that apparently violate the Eddington limit for known compact object masses. However, the bulk of the population of X-ray binaries are low luminosity sources that are difficult to observe in other galaxies. But these sources are important for our understanding of the formation of a whole population of X-ray binaries. To understand their formation processes and compare simulations to observations it is important to know how many sources there are in a galaxy. The shape of the observable luminosity functions, a relatively steep power law, indicates that there must be a cutoff or turnover at low luminosities.

Even with the sensitivity of Chandra and XMM the low luminosity end is only observable in the Milky Way and very nearby galaxies within the Local Group. Despite the observational difficulties there are indications that the lower end of the luminosity function is (almost) in the range of current observatories.

Shtykovskyi \& Gilfanov (2005) have used XMM-Newton observations of HMXBs in the Large Magellanic Cloud. At the lower end of the observable luminosity range at $\sim 10^{34} \mathrm{erg} \mathrm{s}^{-1}$ the number of HMXBs and candidate HMXBs is below the expectation from the universal luminosity function for HMXBs. A possible explanation for this lack of HMXBs is the disappearance of HMXBs due to the "propeller effect". This effect was predicted by Illarionov \& Sunyaev (1975) for accreting, young neutron stars. In these systems, which are only HMXBs due to the age requirement for the neutron star, the magnetic field in conjunction with the rapid rotation of the young neutron star is able to expel the matter that tries to accrete for certain parameter combinations of spin period and field strength. For realistic assumptions about spin periods and magnetic field strengths for HMXBs the "propeller effect" would prevent HMXBs from emitting $\mathrm{X}$-rays in a luminosity range from $10^{32}-10^{34} \mathrm{erg} \mathrm{s}^{-1}$. At the higher luminosities the expected change in the luminosity function is only gradual. Therefore there are at present no definite conclusions possible. 


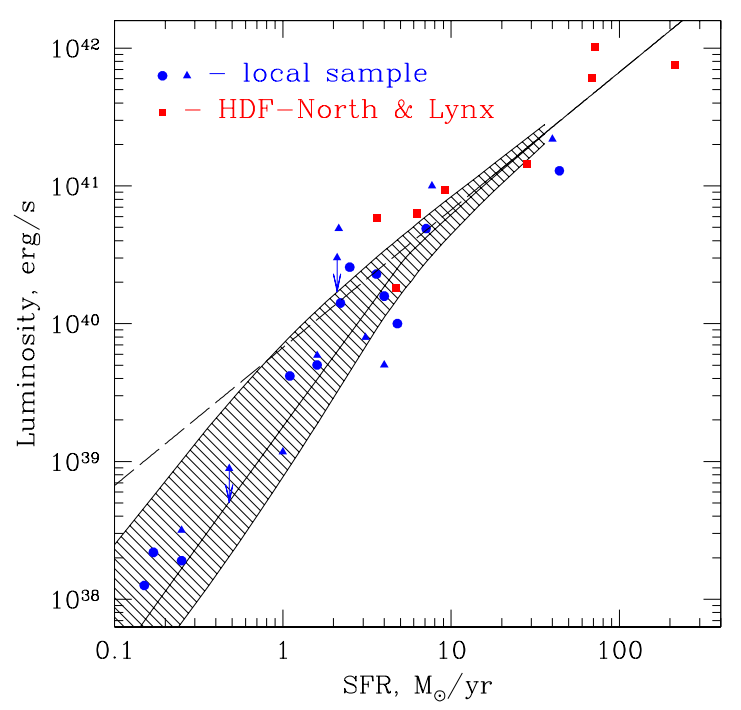

Figure 4. Total X-ray luminosity versus star formation rate. The filled circles and triangles are local galaxies, the filled circles galaxies with resolved X-ray binary populations. The squares are galaxies from Chandra observations of the Hubble Deep Field-North. The solid line is the predicted relation from the universal luminosity function for HMXBs. The shaded area shows the asymmetric 1 sigma uncertainty range.

The "propeller effect" applies only to young neutron stars, i.e. only some HMXBs. McClintock et al. (2004) have observed various transient X-ray binaries in quiescence. The observations reveal a clear distinction between neutron stars and black holes. Neutron stars have quiescent luminosities of $10^{32}-10^{34} \mathrm{erg} \mathrm{s}^{-1}$, whereas black holes have quiescent luminosities of $10^{30}-10^{32} \mathrm{erg} \mathrm{s}^{-1}$. The luminosity is correlated with the orbital period of the system. One of the questions raised by these observations is whether to include quiescent systems into the luminosity function at all.

\section{Integrated properties}

Despite the angular resolution achievable with current X-ray missions the vast majority of galaxies will always be unresolvable. However, it is possible to use the integrated measurements as well to study the relation between X-rays and star formation. The shape of the luminosity function results in a unique relation between the total X-ray flux and star formation rate. In this case some care has to be taken to ensure that the X-ray emission is not related to an AGN or other processes. For sufficiently distant galaxies redshifting ensures that the emission will not be related to supernova remnants and similar low energy emitters.

Fig. 4 shows the relation between star formation rate and X-ray emission for a sample of nearby galaxies where X-ray source populations are resolved and some distant galaxies observed in the Hubble Deep Field-North. There is clearly a non-linear relation at low $\mathrm{SFR} / \mathrm{X}$-ray luminosities, and only at a few $10^{40} \mathrm{erg} \mathrm{s}^{-1}$ the relation changes and becomes linear. The non-linear behaviour is the result of the shape of the luminosity function and its dependence on the star formation rate (Gilfanov et al. 2004). For the luminosity function it is interesting that the shape and break of the total X-ray luminosity versus star formation rate relation depend on parameters of the luminosity function, namely the 
slope and the cutoff luminosity. Obviously the normalization of the luminosity function is the main factor in the numerical constant of the relation. Thus all important parameters of the luminosity function can in principle be determined just from the total X-ray emission of a galaxy. Especially with respect to the cutoff this is important at the present stage. The cutoff is not strongly required from the observed luminosity functions alone. However, the existence of a linear regime in the relation between total X-ray luminosity and star formation rate requires the existence of such a cutoff. If the cutoff did not exist, the relation would be non-linear for all luminosities/star formation rates! The slope $\beta$ of the non-linear part is defined by the slope $\alpha$ of the luminosity function,

$$
\beta=\frac{1}{\alpha-1},
$$

and the break luminosity $L_{\text {tot,break }}$ is defined by the slope and the cutoff luminosity $L_{c u t}$,

$$
L_{\text {tot }, \text { break }} \approx \begin{cases}\frac{L_{\text {cut }}}{2-\alpha} & \text { if } 1<\alpha<2 \\ \frac{L_{\text {cut }}}{\alpha-2} \times\left(\frac{L_{c u t}}{L_{1}}\right)^{\alpha-2} & \text { if } \alpha>2 .\end{cases}
$$

Also the nature of ULXs could possibly be investigated with integrated properties of galaxies. One explanation for the ULX phenomenon is that these objects contain intermediate mass black holes with masses up to $\sim 10000 \mathrm{M}_{\odot}$. Since these objects cannot be the result of stellar evolution processes like neutron stars and stellar mass black holes their luminosity function is different from the one for "normal" X-ray binaries. They are however correlated with star formation because most ULXs are observed in star forming galaxies. If intermediate mass black holes exist and form a different sub-population at high star formation rates, there might be a deviation from the linear regime in the X-ray luminosity/SFR diagram at very high star formation rates.

\section{References}

Cheng, K. P., Collins, N., Angione, R., et al. 1997, UITVi; Vol. U

Gilfanov, M., Grimm, H.-J., \& Sunyaev, R. 2004, MNRAS 351, 1365

Grimm, H.-J., Gilfanov, M., \& Sunyaev, R. 2003, MNRAS 339, 793

Grimm, H.-J., Gilfanov, M., \& Sunyaev, R. 2002, A\&A 391, 923

Hippelein, H., Haas, M., Tuffs, et al. 2003, A\& $A$ 407, 137

Illarionov, A. \& Sunyaev, R. 1975, A\&A 39, 185

McClintock, J., Narayan, R., \& Rybicki, G. 2004, ApJ 615, 402

Shtykovskyi, P. \& Gilfanov, M. 2005, MNRAS 362, 879

\section{Discussion}

PAKULL: Nearby dwarf galaxies tend to have either zero HMXBs or one, which is a ULX, and no lower luminosity sources (down to $\sim 10^{37} \mathrm{erg} \mathrm{s}^{-1}$ ). The sample includes IZw18, the lowest metallicity galaxy there is. Don't you think that another parameter, possibly metallicity, plays an important role in the formation of ULXs, as mass loss rates from massive stars decrease with metallicity.

GRIMM: Metallicity is probably one of the more important parameters affecting primary masses. However, the low number of such sources and other sources of errors in the luminosity function - star formation relation do not allow to distinguish effects of metallicity on the luminosity function at present. 